https://doi.org/10.15407/ujpe64.11.998

B. NOVOSYADLYJ ${ }^{1,2}$

${ }^{1}$ Astronomical Observatory of Ivan Franko National University of Lviv, (8, Kyryla i Methodia Str., Lviv 79005, Ukraine ; e-mail: bohdan.novosyadlyj@lnu.edu.ua)

2 College of Physics and International Center of Future Science of Jilin University (Qianjin Street 2699, Changchun, 130012, P. R. China)

\title{
DARK SECTOR IN COSMOLOGY: DARK ENERGY ON COSMOLOGICAL AND ASTROPHYSICAL SCALES ${ }^{1}$
}

\begin{abstract}
The properties and observational manifestations of the dynamical dark energy on the cosmological and astrophysical scales are discussed. We consider the dynamical dark energy in the form of quintessential and phantom scalar fields with different parameters of the equation of state and the effective sound speed. The evolution of the dynamical dark energy and its impact on the dynamics of expansion of the Universe, halos, and voids, and its behavior in the static gravitational fields of astrophysical objects are analyzed. The current state and possible tests designed to establish the nature of dark energy are highlighted.

Keywords: cosmological model, dark energy, accelerated expansion of the Universe, halos, voids, galaxies.
\end{abstract}

\section{Introduction}

Extragalactic and cosmological observational data collected up to now and interpreted in the framework of current physical theories certify that about $70 \%$ of the energy-mass content of our world is the dark energy which fills the Universe almost uniformly and accelerates its expansion. Its physical nature is still unknown because of its "darkness" and "fingerprints" exclusively on the cosmological scale. About $25 \%$ of the energy-mass content of the Universe consist of dark matter, only about $5 \%$ is usual matter which is described by the Standard Model of particle physics, and the rest is a hidden component of the unknown nature. So, as we can state now that more than $95 \%$ of the content of our Universe is absolutely dark, does not participate into the electromagnetic and strong interactions, and manifests itself by the gravitational action only on the usual matter on the cosmological and astrophysical scales. These two components, dark energy and dark matter, compose the dark sector of cosmology. Establishing the nature of these components becomes extremely important for the elaboration of the physics of galaxies and clusters, cosmology, and particle physics beyond the Standard Model. The totality of the observed data indicates that the most

(C) B. NOVOSYADLYJ, 2019 likely candidate for dark matter is massive collisionless fundamental particles which do not participate in the electromagnetic and strong interactions. Among several ideas about the nature of dark energy discussed in the literature, the hypothesis that it is a scalar field with a violated weak or null energy condition seems the most promising in terms of the possibility to be tested by comparison of theoretical predictions with observational data. The scalar field can be quintessential dark energy, phantom or changing type from one to another (quintom) at different moments of time, or be vacuum-like (or $\Lambda$-type) dark energy. Here, we will analyze the behavior, properties, and possible observational manifestations of dynamical dark energy on the cosmological and astrophysical scales.

\section{Scalar Field as Dark Energy}

Cosmologists have enough observational evidence that our Universe is expanding with positive acceleration, instead of deceleration expected for a world filled with ordinary matter and described in the framework of general relativity. The physical essence which

1 This work is based on the results presented at the XI BolyaiGauss-Lobachevskii (BGL-2019) Conference: Non-Euclidean, Noncommutative Geometry and Quantum Physics.

ISSN 2071-0194. Ukr. J. Phys. 2019. Vol. 64, No. 11 
causes such unusual dynamics of the whole world was called "dark energy" [1]. From the time of the first reliable observational proof of its existence in 1998 [2-4] the dozens of thousands papers are devoted to the analysis of different models of dark energy (see books and few review articles [5-9] for example). One of the most developed models in the terms of comparing of numerical predictions with observational data and the determination of the parameters and their confidence intervals is a scalar field that fills the Universe almost homogeneously and slowly rolls down to the minimum of its own potential in the case of a quintessential scalar field or slowly rolls up to the maximum in the case of a phantom one. Here, we try to elucidate the main properties and manifestations of the scalar field dark energy in the cosmological background with the Friedmann-LemaîtreRobertson-Walker (FLRW) metric, in the halos and voids, in the static world with the Minkowski metric, and in the vicinity of compact spherical bodies with the Schwarzschild metric. There are many possible realizations of such fields, that is why the additional observational or experimental tests, which could help to constrain the number of candidates at least in the class of scalar field models of dark energy are needed.

The scalar field dark energy can be set by the Lagrangian $\mathcal{L}(X, U)$ with a kinetic term $X \equiv \partial_{i} \varphi \partial^{i} \varphi / 2$ and a given potential $U(\varphi)$ or by the energymomentum tensor $T_{i j}\left(\rho_{\mathrm{de}}, p_{\mathrm{de}}\right)$ in the phenomenological approach. In the last case, the dark energy is assumed, as usual, to be the perfect fluid described by a smallest number of parameters: the density in units of the critical one at the current epoch $\Omega_{\mathrm{de}} \equiv \rho_{\mathrm{de}}^{0} / \rho_{\mathrm{cr}}^{0}$, the parameter $w_{\text {de }} \equiv p_{\text {de }} / \rho_{\text {de }}<-1 / 3$ of the equation of state (EoS), and the square of effective sound speed $c_{s}^{2} \equiv \delta p_{\mathrm{de}} / \delta \rho_{\mathrm{de}}>0$. The variables of the phenomenological approach are connected with the scalar field Lagrangian by the known relations:

$$
\begin{aligned}
& \rho_{\mathrm{de}}=2 X \mathcal{L}_{, X}-\mathcal{L}, \quad p_{\mathrm{de}}=\mathcal{L}, \\
& w_{\mathrm{de}}=\frac{\mathcal{L}}{2 X \mathcal{L}_{, X}-\mathcal{L}}, \quad c_{s}^{2}=\frac{\mathcal{L}_{, X}}{2 X \mathcal{L}_{, X X}+\mathcal{L}_{, X}},
\end{aligned}
$$

where $\mathcal{L}_{, X} \equiv \partial \mathcal{L} / \partial X$. The phenomenological approach is convenient for practical calculations and putting the observational constraints on model parameters. However, it gives very little information about the physical nature of dark energy. On the other hand, the scalar field approach is well suited for the study of the physics of dark energy, but is not as usable in practice as the former one. So, it is often necessary to combine both methods of modeling of dark energy and to use the phenomenological perfect fluid in numerical calculations, while studying the physical features of scalar fields reconstructed to mimic the behavior of this perfect fluid. For example, assuming that $c_{s}^{2}=$ cont, we obtain the following general form of the Lagrangian from (1):

$$
\begin{aligned}
\mathcal{L} & =V X^{\alpha}-U, \quad U=\frac{c_{s}^{2}-w_{\mathrm{de}}}{1+c_{s}^{2}} \rho_{\mathrm{de}} \\
V & =V_{0}\left(w_{\mathrm{de}}-c_{s}^{2}\right) \rho_{\mathrm{de}} \\
X & =\left[\frac{1}{V_{0}} \frac{c_{s}^{2}}{1+c_{s}^{2}} \frac{1+w_{d e}}{w_{\mathrm{de}}-c_{s}^{2}}\right]^{\frac{1}{\alpha}},
\end{aligned}
$$

where $\alpha \equiv\left(1+c_{s}^{2}\right) /\left(2 c_{s}^{2}\right)$, and $V_{0}$ is an arbitrary integration constant. When $c_{s}^{2}=1$, Lagrangian (2) becomes the canonical one: $\mathcal{L}= \pm X-U$. We will analyze the properties of the scalar field dark energy with such Lagrangian at the non-stationary cosmological background, as well as in the stationary space-time of galaxies and spherical static objects. We present the space-time metric in the form

$d s^{2}=e^{\nu(t, r)} d t^{2}-e^{\lambda(t, r)} d r^{2}-r^{2} e^{\mu(t, r)} Y(\theta, \varphi)$,

where $e^{\lambda(t, r)}=e^{\mu(t, r)}=a^{2}(t)$ and $\nu(t, r)=0$ for the cosmological background $(a(t)$ is a scale factor), $\nu(t, r)=\lambda(t, r)=\mu(t, r)=0$ for the static Minkowski world and $e^{\nu(r)}=e^{-\lambda(r)}=1-r_{g} / r$ and $\mu=0$ in the vicinity of a spherical static object with mass $M, r_{g} \equiv$ $\equiv 2 G M / c^{2}$ is the gravitational radius, and $Y(\theta, \varphi)=$ $=\left(d \theta^{2}+\sin ^{2} \theta d \varphi^{2}\right)$.

The dependence of the dark energy density on the time or scale factor $a$ is obtained by the integration of the continuity equation $T_{0 ; i}^{i}=0$ in the world with metric (3).

For the non-stationary homogeneous isotropic FLRW world, the density has the general form for any dependence of the EoS parameter $w_{d e}$ on the scale factor: $\rho_{\mathrm{de}}=\rho_{\mathrm{de}}^{(0)} a^{-3\left(1+\tilde{w}_{\mathrm{de}}\right)}$ with $\tilde{w}_{\mathrm{de}}=(\ln a)^{-1} \times$ $\times \int_{1}^{a} w_{\mathrm{de}}(a) d \ln a$. To specify $w_{\mathrm{de}}(a)$, we assume that $c_{a}^{2} \equiv \dot{p}_{\mathrm{de}} / \dot{\rho}_{\mathrm{de}}=$ const (overdot denotes the derivative w.r.t. time $t$ ), which gives the analytic dependences of $w_{\mathrm{de}}$ and $\rho_{\mathrm{de}}$ on $a[10,11]$ :

$$
\begin{aligned}
& w_{\mathrm{de}}=\frac{\left(1+c_{a}^{2}\right)\left(1+w_{0}\right)}{1+w_{0}-\left(w_{0}-c_{a}^{2}\right) a^{3\left(1+c_{a}^{2}\right)}-1,} \\
& \rho_{\mathrm{de}}=\rho_{\mathrm{de}}^{(0)} \frac{\left(1+w_{0}\right) a^{-3\left(1+c_{a}^{2}\right)}+c_{a}^{2}-w_{0}}{1+c_{a}^{2}},
\end{aligned}
$$



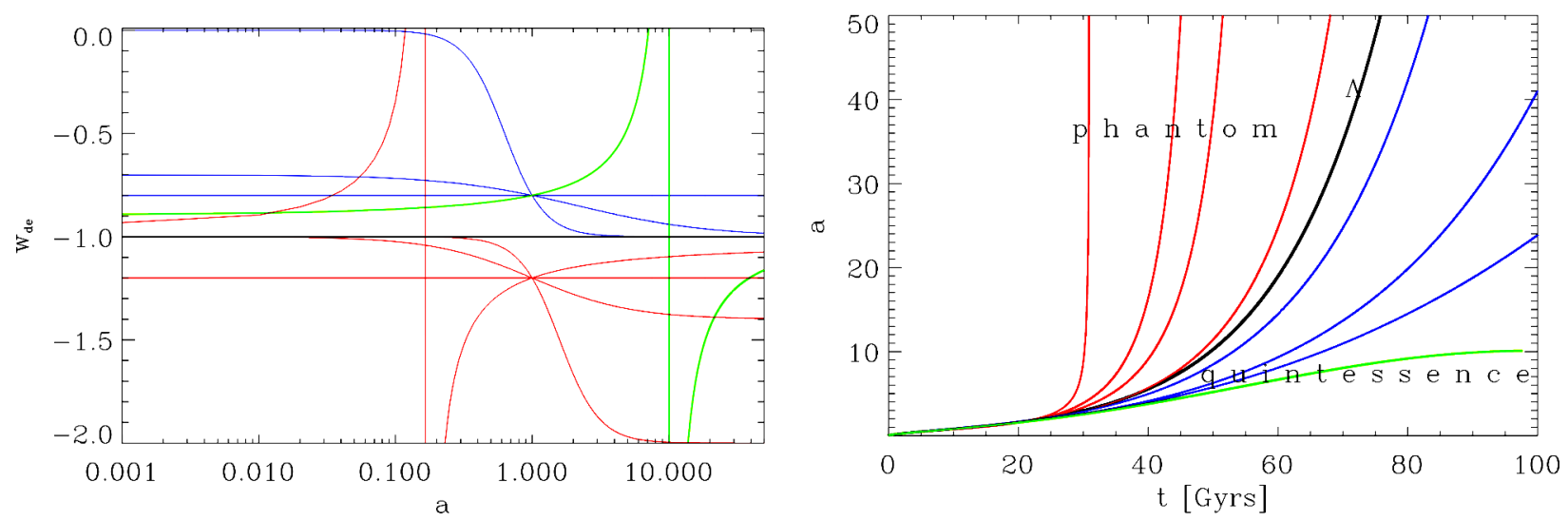

Fig. 1. Left: $w_{\mathrm{de}}(a)$ in the expanding Universe for different values of $w_{0}$ and $c_{a}^{2}$. Right: $a(t)$ for cosmological models with the scalar field dark energy and $w_{\mathrm{de}}(a)$ from the left panel. $\left(H=70 \mathrm{~km} / \mathrm{s} / \mathrm{Mpc}, \Omega_{m}=0.3, \Omega_{\mathrm{de}}=0.7\right.$ and different sets of values $\left(w_{0}, c_{a}^{2}\right)$ : red lines $-(-1.2,-2.0),(-1.2,-1.4),(-1.2,-1.2),(-1.2,-1.03)$, black line $-(-1.0,-1.0)$, blue lines $-(-0.8,-0.0),(-0.8$, $-0.7),(-0.8,-0.8)$, and green line $-(-0.8,-0.9)$

where $w_{0}$ is the EoS parameter at the current epoch $(a=1)$. For such scalar field, its phenomenological density $\rho_{\text {de }}$ and pressure $p_{\text {de }}$ are analytic functions of $a$ for any values of the constants $c_{a}^{2}$ and $w_{0}$ defining the type and the dynamics of the scalar field. Both have the clear physical meaning: $w_{0}$ is the EoS parameter $w_{\text {de }}$ at the current epoch, $c_{a}^{2}$ is the asymptotic value of the EoS parameter $w_{\text {de }}$ at early times $(a \rightarrow 0)$ for $c_{a}^{2}>-1$ and in the far future $(a \rightarrow \infty)$ for $c_{a}^{2}<-1$. The asymptotic value of $w_{\text {de }}$ in the opposite time direction is -1 in both cases. The examples of evolution tracks of the EoS parameter $w_{\mathrm{de}}$ for different values of $w_{0}$ and $c_{a}^{2}$ are presented in Fig. 1. The energy density $\rho_{\mathrm{de}}$ and pressure $p_{\text {de }}$ are smooth monotonic functions of $a$ for all relations between $c_{a}^{2}$ and $w_{0}$, while $w_{\text {de }}$ has a discontinuity of the second kind in the case $w_{0}<c_{a}^{2}<-1$ and $-1<c_{a}^{2}<w_{0}<0$, when the scalar field energy density passes through zero. So, the Lagrangian of such scalar field model of dark energy can be reconstructed accurately up to a constant $V_{0}$, if the parameters $\Omega_{\mathrm{de}}$, $w_{0}, c_{a}^{2}$ and $c_{s}^{2}$ are given or determined using observational data.

For the stationary Minkowski or Schwarzschild world and the scalar field dark energy with $p_{\text {de }}(r) /$ $/ \rho_{\mathrm{de}}(r)=w_{\mathrm{de}}(r)<0$ and $c_{s}^{2}=$ const $>0$, one can obtain [12]:

$w_{\mathrm{de}}=c_{s}^{2}-\left(c_{s}^{2}-\bar{w}_{\mathrm{de}}\right) \bar{\rho}_{\mathrm{de}} / \rho_{\mathrm{de}}(r)$,

where $\bar{\rho}_{\text {de }}$ and $\bar{w}_{\text {de }}$ are background values of the density and EoS parameters. As it follows from Eq. (2), the kinetic term and potential are:

$$
\begin{aligned}
& X=\left[\frac{c_{s}^{2}}{V_{0} \bar{\rho}_{d e}}\left(\frac{\bar{\rho}_{d e}}{1+c_{s}^{2}}+\frac{\rho_{d e}}{\bar{w}_{d e}-c_{s}^{2}}\right)\right]^{\frac{1}{\alpha}}, \\
& U=\frac{c_{s}^{2}-\bar{w}_{d e}}{1+c_{s}^{2}} \bar{\rho}_{d e} .
\end{aligned}
$$

Thus, a perfect fluid with a state equation (5) can be a static scalar field with a constant potential and a density-dependent kinetic term.

Phantom dark energy with a negative kinetic term has unusual properties and causes a Cosmic Doomsday [13]. Up to now we have no physical motivation to ban the fields with a negative kinetic term in their Lagrangians. Perhaps, a deep analysis of their microphysical properties and their effect on the quantum stability of vacuum will give such limitations. It was shown $[14,15]$ that minimally coupled scalar fields with a linear negative kinetic term may cause a UV quantum instability of the vacuum manifesting itself in the production of pairs of ghosts, photons or gravitons as a consequence of the violation of the null energy condition. It can be prevented by introducing the squared kinetic term in the Lagrangian as in the ghost condensate model $[16,17]$ or by second derivatives of the scalar field as in the kinetic braiding scalar-tensor model [18]. For the late type phantom scalar fields the produced ghosts typically carry low energy, so, their decay rates are strongly timedilated. On the other hand, the time scale of this instability for phantom dark energy can be much larger 
than the cosmological one, making this effect unsuitable for constraining the parameters of the model at the present level of observations. This is why we concentrate our attention here on the classical properties of scalar field models of dark energy and on the possibilities to determine their parameters by comparison of predictions with available observational data.

\section{Accelerated Expansion} of the Universe and $d_{L}-z$ and $d_{A}-z$ Cosmological Tests

We consider a multicomponent model of the Universe filled by matter (cold dark matter and baryons) with density $\Omega_{m}$, relativistic particles (thermal electromagnetic radiation and massless neutrino) with density $\Omega_{r}$, and a scalar field as described above. The background Universe is assumed to be spatially flat, homogeneous, and isotropic with the FLRW metric. The dynamics of expansion of the Universe can be deduced from the Einstein equations

$R_{i j}-\frac{1}{2} g_{i j} R=8 \pi G\left(T_{i j}^{(m)}+T_{i j}^{(r)}+T_{i j}^{(\mathrm{de})}\right)$,

where $R_{i j}$ is the Ricci tensor, and $T_{i j}^{(m)}, T_{i j}^{(r)}, T_{i j}^{(\mathrm{de})}$ are the energy-momentum tensors of non-relativistic matter $(m)$, relativistic matter $(r)$, and dark energy (de), respectively. Assuming that the interaction between these components is only gravitational, each of them satisfies the differential energy-momentum conservation law separately: $T_{j ; i}^{i(N)}=0$. Einstein's equations together with the conservation law equations lead to the Friedmann equations, which describe the rate and acceleration of expansion of the Universe:

$$
\begin{aligned}
& H=H_{0} \sqrt{\Omega_{r} / a^{4}+\Omega_{m} / a^{3}+\Omega_{\mathrm{de}} f(a)}, \\
& q=\frac{1}{2} \frac{2 \Omega_{r}+\Omega_{m} a+\left(1+3 w_{\mathrm{de}}\right) \Omega_{\mathrm{de}} a^{4} f(a)}{\Omega_{r}+\Omega_{m} a+\Omega_{\mathrm{de}} a^{4} f(a)},
\end{aligned}
$$

where $f(a)=\rho_{\mathrm{de}}(a) / \rho_{\mathrm{de}}(1)$. Here, $H \equiv \dot{a} / a$ is the Hubble parameter (expansion rate), $q \equiv-a \ddot{a} / \dot{a}^{2}$ is the deceleration parameter. Equations (7) completely describe the dynamics of expansion of the homogeneous and isotropic Universe. In the past, it was dominated by radiation and matter. Now and in the distant future, it is dominated by the scalar field dark energy. The integration of the first Friedmann equation (7) gives us the dependence $a(t)$ shown in the right panel of Fig. 1 for the scalar field dark energy with different values of $w_{0}$ and $c_{a}^{2}$. The positive cosmological constant, or a special case in our model where $w_{0}=c_{a}^{2}=-1$, will lead to the exponential external expansion $a \propto e^{H t}$ in the far future (dark line marked by $\Lambda$ ) which is called the late inflation or late de Sitter expansion. The quintessence scalar field dark energy will lead to the power-law quaside Sitter expansion (blue lines). The models with $-1<c_{a}^{2}<w_{0}<-1 / 3$ will expand to the turn around a point and then re-collapse (green line) to the Big Crunch singularity. When $c_{a}^{2}<w_{0}<-1$, we have the phantom scalar field dark energy which will lead to the superfast expansion of the Universe (red lines). In this case, the $a$-infinity is reached within a finite time $t_{\mathrm{BR}}-t_{0} \approx H_{0}^{-1}\left|1+c_{a}^{2}\right|^{-1} \sqrt{\left(1+c_{a}^{2}\right) /\left(1+w_{0}\right) / \Omega_{\mathrm{de}}}$, which is called as a Big Rip singularity [13].

In the FLRW space-time, the luminosity distance to the source at a redshift $z$ with the known luminosity $L$ and measured flux $F$, defined as $d_{L} \equiv \sqrt{L / 4 \pi F}$, and the angular diameter distance to the source at a redshift $z$ with the known linear diameter $D$ and measured angular diameter $\Theta$, defined as $d_{A} \equiv D / \Theta$, in the models with given cosmological parameters and dark energy model are as follows:

$d_{L}=(1+z) \int_{0}^{z} \frac{d z^{\prime}}{H\left(z^{\prime}\right)}, \quad d_{A}=\frac{1}{1+z} \int_{0}^{z} \frac{d z^{\prime}}{H\left(z^{\prime}\right)}$,

where $z \equiv a^{-1}-1$. The relation $d_{L}-z$ for "standard candles" and $d_{A}-z$ for "standard meters" are the wellknown cosmological tests to realizations of which a lot of efforts of two generations of cosmologists and astrophysicists have been devoted. The first successful realization of $d_{L}-z$ for Ia Supernovae (SNe Ia) have been done in [2-4] which was the first observational evidence for the dark energy existence. Thenceforth the both cosmological tests have been realized for different redshifts, classes of objects, and ranges of electromagnetic radiation. Dozens of independent measurements supported this discovery and constrained the dark energy parameters (see the above cited reviews) $\Omega_{\mathrm{de}}$ and $w_{\mathrm{de}}$. For the constraining of $c_{s}^{2}$, the density perturbations of dark energy must be taken into account in the computations of the formation of a large-scale structure. Since the amplitudes of scalar field dark energy perturbations are essentially lower than dark matter ones on all scales and in all epochs $[10,11]$, the effect is too small to constrain $c_{s}^{2}$ by current observational data [19]. 
The best-fit values and $2 \sigma$ confidence limits (C.L.) for parameters of scalar field dark energy, $w_{0}, c_{a}^{2}$, and $c_{s}^{2}$

\begin{tabular}{|c|c|c|c|}
\hline$\Omega_{\mathrm{de}}$ & $w_{0}$ & $c_{a}^{2}$ & $c_{s}^{2}$ \\
\hline $0.71 \pm 0.025$ & $-1.13 \pm 0.13$ & $-1.33 \pm 0.25$ & $0.5 \pm 0.5$ \\
\hline
\end{tabular}

In the recent papers, to determine the best-fit values and confidence ranges of the cosmological parameters and the parameters of dark energy, we have used the data on the angular power spectra of cosmic microwave background temperature fluctuations (WMAP9 and Planck) [20,21], Hubble constant measurement (HST) [22], baryon acoustic oscillations (BAO) [23-25] and SNe Ia luminosity distances (SNLS3 and Union2.1 compilation) [26, 27]. We apply a Markov Chain Monte Carlo routine CosmoMC [28] to map out the likelihood in the multidimensional parameter space. The most reliable determination of the dark energy parameters are obtained from the Planck $+\mathrm{HST}+\mathrm{BAO}+\mathrm{SNLS} 3$ dataset [19] and are presented in the Table. These results are in agreement with other determinations [29-32].

\section{Dark Energy in the Halos and Voids}

To analyze the behavior of dynamical dark energy in the formation of halo and voids, the evolution of spherical density and velocity perturbations in the four-component medium (dark matter, dark energy, baryonic matter, and radiation) should be considered from the linear superhorizon stage (when wave number $k$ of Fourie mod of perturbation is $\ll 1 / c t$ ) in the radiation-dominated epoch to the nonlinear subhorizon one $(k \gg 1 / c t)$ in the current epoch. For this purpose, a semianalytic model based on the integration of the system of partial differential equations describing the evolution of perturbations is used. Numerical solutions for the density and velocity perturbations of dark matter and dark energy allowed us to assess the influence of the latter on the development of elements of the large-scale structure of the Universe. Such approach is described in details in our papers [33-36].

Up to now, it is established that the dynamics of the quintessential dark energy at the center of halo or void of dark matter strongly depends on the value of the effective sound speed. Figure 2 illustrates the formation of a dark matter halo (solid line) and the dynamics of dark energy (dashed line) in the terms of density and velocity perturbations in the reference system which is comoving to the cosmological background. When $c_{s} \sim 1$, the dark energy (type of classical scalar field) is only slightly perturbed, and its density is practically the same as the density on the cosmological background (Fig. 2, left). Dark energy with a small effective sound speed $\left(c_{s} \ll 1\right)$ is an important dynamic component of the halo: it is capable of accretion into the formed halo of dark matter, by affecting the value of its finite parameters (Fig. 2, right) [33]. After the virialization of a dark matter halo (in Fig. 2 , it is at $\left.a_{\mathrm{vir}} \approx 0.032\left(z_{\mathrm{vir}} \approx 30\right)\right)$, the matter density $\rho_{m}^{\mathrm{vir}}=$ const, the matter density perturbation $\delta_{m}^{\mathrm{vir}} \sim a^{3}$, since $\delta_{m} \equiv \rho_{m} / \bar{\rho}_{m}-1$, and the peculiar velocity $v_{m}$ equals the Hubble one with opposite sign, or $V_{m}^{\text {vir }} \equiv v_{m} / H r=-1$. On the base of studying of formation of single spherical halos in the threeor four-component Universe in $[33,36]$, we concluded that physical and statistical properties of the most massive earliest virialized structures should discriminate the dark energy with $c_{s} \sim 1$ and $c_{s} \sim 0$. They are now under intense observational and numerical simulation investigations and soon will shed more light also on the nature of dark energy.

The giant voids in the large-scale structure of the Universe can be useful for the determination of dark energy parameters too. Really, the ratio of dark energy density to matter one (dark matter plus baryons),

$\frac{\rho_{\mathrm{de}}^{\mathrm{void}}}{\rho_{m}^{\text {void }}}=\frac{1+\delta_{\mathrm{de}}^{\mathrm{void}}}{1+\delta_{m}^{\text {void }}} \frac{\Omega_{\mathrm{de}}}{\Omega_{m}}$,

is largest in the voids, since $-1<\delta_{m}^{\text {void }}<0$ and $\left|\delta_{m}^{\text {void }}\right| \gg\left|\delta_{\mathrm{de}}^{\text {void }}\right|$ there. For the real voids with $\delta_{m}^{\text {void }} \approx$ $\approx-(0.8-0.9)$ in the cosmology with $\Omega_{m} \approx 0.3$ and $\Omega_{\mathrm{de}} \approx 0.7$, the ratio is $\approx 12-23$, while, at the cosmological background, it is 2.3 .

The formation of giant voids from the initial adiabatic cosmological perturbations of the space-time metric, density, and velocity of matter in a cosmological model with dynamical dark energy have been analyzed in $[34,35]$ (see also references therein). It was shown that the negative density perturbations with the initial radius of about $50 \mathrm{Mpc}$ in the reference system comoving with the cosmological background and the amplitude corresponding to the r.m.s. temperature fluctuations of the cosmic microwave background lead to the formation of voids with the matter density contrast up to -0.9 , max-

ISSN 2071-0194. Ukr. J. Phys. 2019. Vol. 64, No. 11 

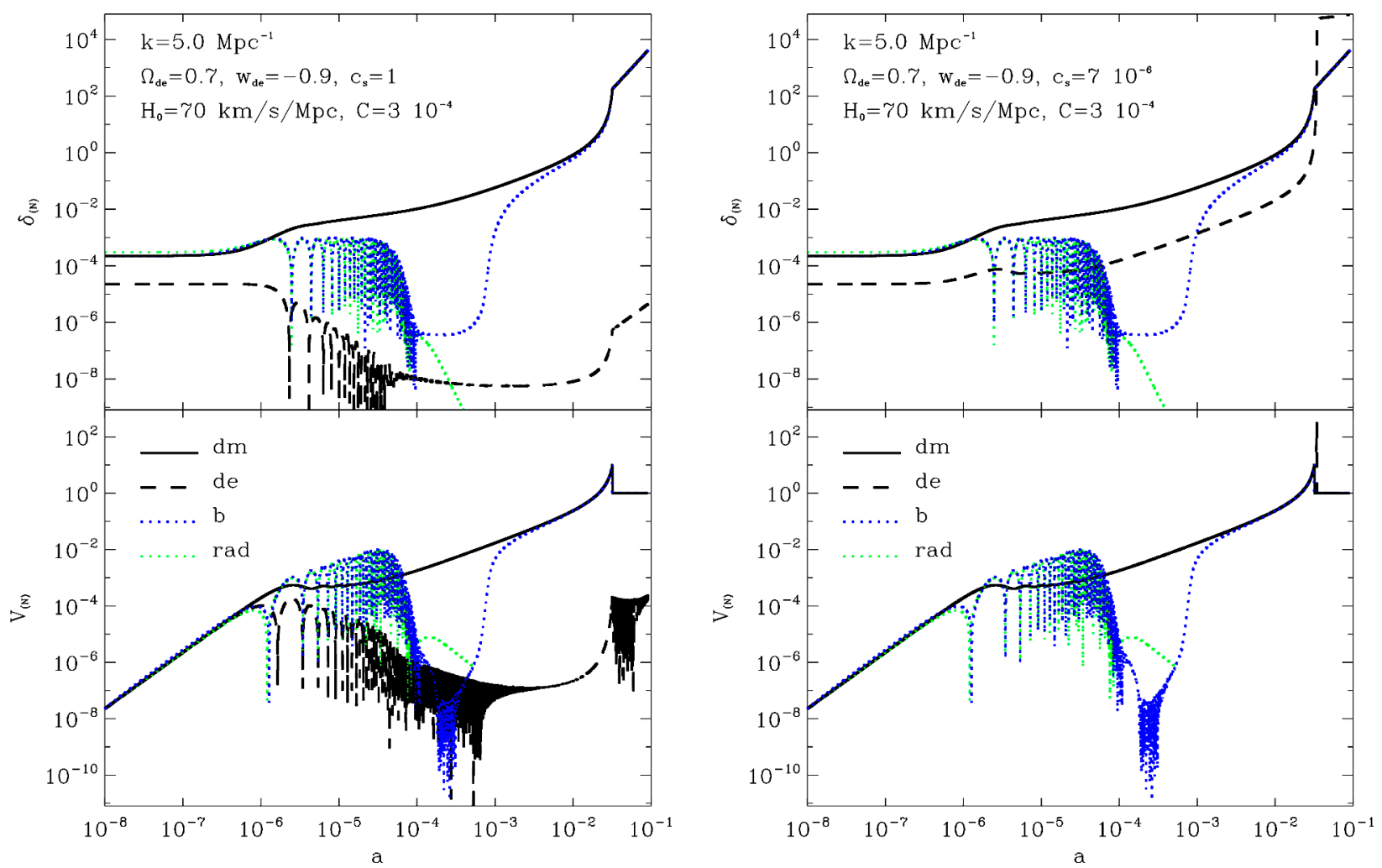

Fig. 2. Evolution of the density and velocity perturbations of matter, dark energy, baryons, and radiation which form the halo with $M \approx 510^{9} \mathrm{M} \odot$. In the left panel, the dark energy is a scalar field with $c_{s}=1$; in the right one, $c_{s} \ll 1$ (From [33, 36])

imal peculiar velocity about $400 \mathrm{~km} / \mathrm{s}$, and the radius in the comoving coordinates close to the initial one. An important feature of the formation of voids from the analyzed initial amplitudes and profiles is the establishment of a surrounding matter overdensity shell. The evolution of dark energy density and velocity perturbations strongly depends on the values of $w_{\mathrm{de}}$ and $c_{s}$. It was shown also that the ratio of the peculiar velocity of matter in units of the Hubble flow to the matter density contrast in the central part of a void is sensitive to the values of dark energy parameters, what is shown in Fig. 3. Hence, measurements of the mass density and peculiar velocities of galaxies in the voids can be used for determination of parameters of dark energy.

\section{Dark Energy in Galaxies}

We suppose that the spacetime in stationary galaxies and clusters is the Minkowski one, and the EoS parameter $w_{\text {de }}$ varies with a variation of the local density of a scalar field $\rho_{\text {de }}$ according to Eq. (5). For the estimation of the background density of dark energy $\bar{\rho}_{\text {de }}$ and matter $\bar{\rho}_{m}$ in the galaxies, one can use the halo model of galaxies and the formation of clusters $[37,38]$. According to this model, the average density of matter after the virialization and the establishment of the dynamical equilibrium is $\rho_{m}^{\mathrm{vir}}=\Delta_{v c} \rho_{\mathrm{cr}}\left(z_{\mathrm{col}}\right)$, where $\Delta_{v c} \approx 100$ in the current epoch, $\approx 150$ at $z=1$ and $\approx 180$ at $z>10$, and the critical density is taken for the moment of the collapse of the central part of a uniform dust-like halo. For the further estimation, we assume that the redshift of the collapse $z_{\text {col }}$ is 20 for a typical galaxy and 1 for a typical cluster of galaxies. The simple estimations (see for details [39]) give the average matter density in typical massive galaxy and rich cluster of galaxies $\rho_{m}^{\text {gal }} \approx 5 \times 10^{-24} \mathrm{~g} / \mathrm{cm}^{3}$, $\rho_{m}^{\mathrm{cl}} \approx 5 \times 10^{-27} \mathrm{~g} / \mathrm{cm}^{3}$.

The scalar field as dark energy, which almost uniformly fills the entire Universe, practically does not participate in the virialization of the dark matter, but it feels changes of the gravitational potential of the halo in the time and space. After the halo (galaxy 

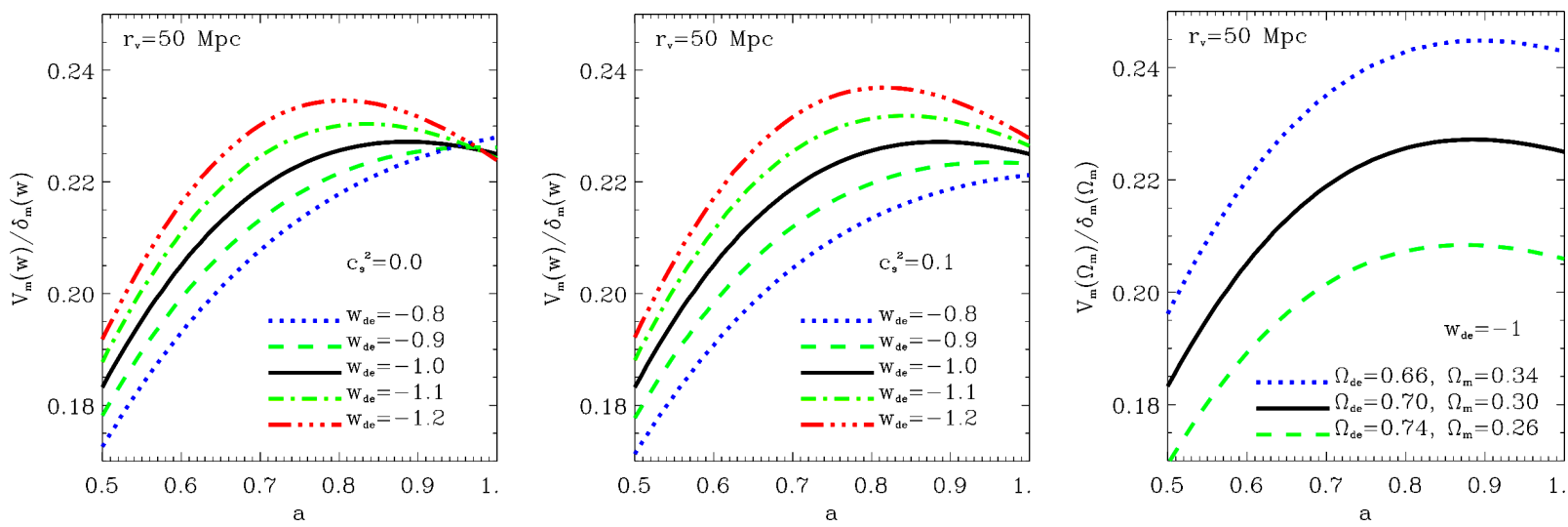

Fig. 3. Ratio $V_{m} /\left|\delta_{m}\right|$ for dynamical dark energy models with different values of the EoS parameter $w_{\text {de }}$, effective sound speed $c_{s}$, and density parameter $\Omega_{\mathrm{de}}$ in the cosmological models with zero curvature. (From [35])

or cluster of galaxies) has been separated from the expansion of the Universe, which happens at the moment $z_{t a}\left(t_{t a}=t_{\mathrm{col}} / 2\right.$ in the Einstein-de Sitter model), the dynamics of dark energy changes according to the dynamics of a local world. When the halo after the virialization is stabilized, the static world in its volume can be considered as the Minkowski one (we ignore here the local inhomogeneities and the space-time curvature). For the estimation, we assume that the dynamics of dark energy in galaxies and clusters stabilizes at their $z_{\mathrm{col}}$, and, from this moment, its density does not change. As $\rho_{\mathrm{de}}(z)=\Omega_{\mathrm{de}} \rho_{\mathrm{cr}}^{(0)}(1+$ $+z)^{3\left(1+w_{\mathrm{de}}\right)}$, for the quintessential scalar field with $w_{\mathrm{de}}=-0.9$ for a galaxy and a cluster of galaxies, we get $\rho_{\mathrm{de}}^{\text {gal }} \approx 2 \times 10^{-29} \mathrm{~g} / \mathrm{cm}^{3}, \rho_{\mathrm{de}}^{\mathrm{cl}} \approx 8 \times 10^{-30} \mathrm{~g} / \mathrm{cm}^{3}$. For the phantom field, respectively, $\rho_{\mathrm{de}}^{\mathrm{gal}} \approx 2.5 \times$ $\times 10^{-30} \mathrm{~g} / \mathrm{cm}^{3}, \rho_{\mathrm{de}}^{\mathrm{cl}} \approx 5.2 \times 10^{-30} \mathrm{~g} / \mathrm{cm}^{3}$. So, in the galaxies and clusters, $\rho_{\mathrm{de}} \ll \rho_{m}$.

Another important question connected with properties of dark energy in a stationary world is addressed to its gravitational instability. Our analysis [39] showed that the scalar field dark energy with Lagrangian (2) and EoS (5) is stable and can only oscillate with constant amplitude on scales smaller than the Jeans scale $\lambda_{J} \approx 2660\left(1+3 c_{s}^{2}\right)^{-1 / 2}(1+$ $\left.+w_{\text {de }}\right)^{-1 / 2}\left(6.4 \times 10^{-30} / \rho_{\text {de }}\right)^{1 / 2} \mathrm{Mpc}$, which is much larger for realistic values of $\rho_{\text {de }}\left(\right.$ in $\left.\mathrm{g} / \mathrm{cm}^{3}\right)$ than the scales of observable structures interesting for astrophysics. The phantom dark energy has no Jeans scale: the perturbations on all scales can only oscillate with a constant amplitude.

In the two-component medium, the amplitudes of the dark matter density perturbations are much larger than the dark energy ones and almost completely determine the gravitational potential of perturbations on the given scale [39]. Dark energy can monotonically flow into gravitational potential wells of positive matter density perturbations $\left(\delta_{m}>0\right)$ oscillating with significantly lower constant amplitude and forming a dark energy overdensity $\left(\delta_{\mathrm{de}}>0\right)$ in the case of quintessential dark energy $\left(-1<w_{\text {de }}<\right.$ $<-1 / 3)$ and dark energy underdensity $\left(\delta_{\mathrm{de}}<0\right)$ in the case of phantom one $\left(w_{\mathrm{de}}<-1\right)$. We should note, however, that the amplitudes of perturbations of dark energy on all galaxy and cluster scales are negligibly small in comparison with amplitudes of perturbations of dark matter $\left(\delta_{\mathrm{de}} \ll \delta_{m}, V_{\mathrm{de}} \ll V_{m}\right)$. Only in the models of dark energy with $c_{s} \rightarrow 0$, the velocity perturbations of dark energy are close to the velocity perturbations of matter: $V_{\mathrm{de}} \rightarrow V_{m}$, while $\delta_{\mathrm{de}} \rightarrow\left(1+w_{\mathrm{de}}\right) \delta_{m}$. If $c_{s} \geq 0.1$, then $V_{\mathrm{de}} \leq 10^{-3} V_{m}$ on the scales of galaxies and $V_{\mathrm{de}} \leq 10^{-2} V_{m}$ on the scales of clusters of galaxies. So, the amplitudes of perturbations of dark energy may be larger in the dynamical models of dark energy with a small value of the speed of sound and may leave traces in the structure on scales of galaxies and clusters of galaxies.

\section{Dark Energy in the Vicinities of Compact Astrophysical Objects}

\subsection{Static distribution}

One more important question regarding dynamical dark energy is connected with its behavior in the strong gravitational fields of massive compact objects in galaxies, e.g., globular clusters, stars, or black holes. It can be formulated as follows: whether is or 

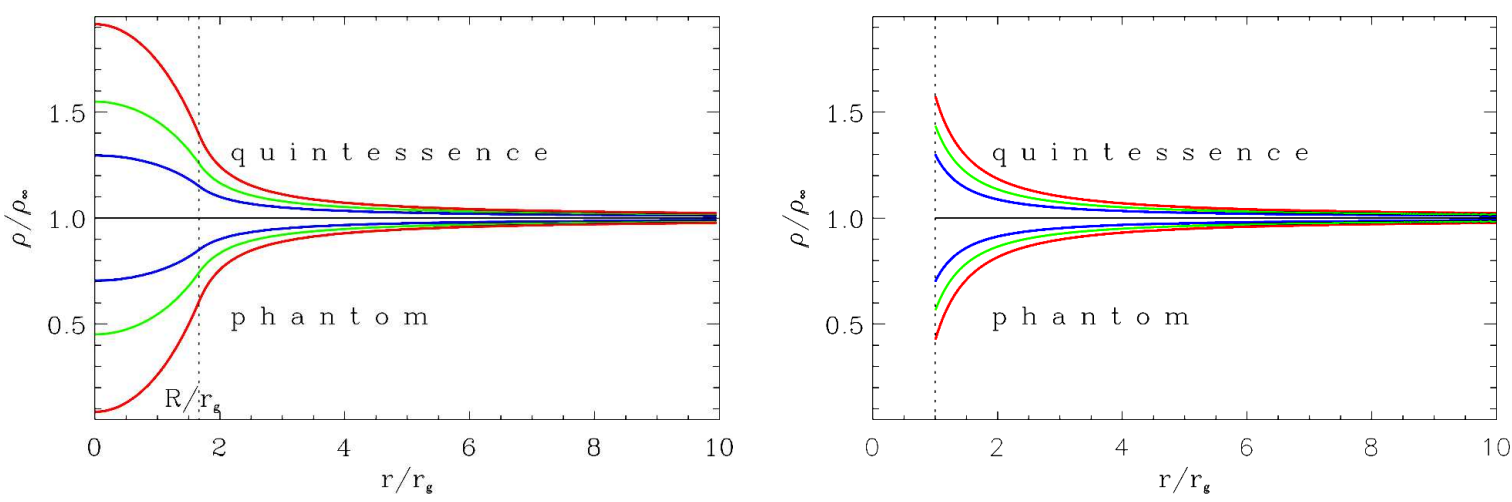

Fig. 4. Dependence of the density of dark energy on the distance from the center of a central body for the quintessence $\left(w_{\infty}=-0.8\right)$ and phantom $\left(w_{\infty}=-1.2\right)$ scalar fields with the effective sound speed $c_{s}^{2}=1$ (blue lines), $2 / 3$ (green lines), $1 / 2$ (red lines) from bottom to top for quintessence dark energy and from top to bottom for phantom dark energy. Left panel: homogeneous spherical object with $R / r_{g}=5 / 3$, distribution under the condition of static equilibrium; right panel: black hole, distribution under the stationary accretion condition

not some possibility for dark energy to be agglomerated in enough amount to have some manifestation in spite of its negligible mean density in galaxies? To answer this question, we have analyzed the dynamics of scalar field with Lagrangian (2) and EoS (5) in the gravitational fields of non-rotational stars and black holes, in whose vicinities the space-time metric is the Schwarzschild one [12].

The first important conclusion of such analysis is the existence of a static solution for dark energy in the gravitational fields of spherical static objects. The equation expressing the static condition is the only nontrivial energy-momentum conservation law equation $T_{1 ; k}^{k}=0$ :

$\frac{d p_{\mathrm{de}}}{d r}+\frac{1}{2}\left(\rho_{\mathrm{de}}+p_{\mathrm{de}}\right) \frac{d \nu}{d r}=0$

where $p_{\text {de }}$ and $\rho_{\text {de }}$ are unknown functions of $r, \nu(r)$ is the metric function for the Schwarzschild space-time (see Eq. (3) and text below of it). For the scalar field with Lagrangian (2) and EoS (5), Eq. (10) can be integrated and gives the dependence of the density of dark energy on the radial coordinate,

$\rho_{\mathrm{de}}(r)=\bar{\rho}_{\mathrm{de}} \frac{c_{s}^{2}-\bar{w}_{\mathrm{de}}+\left(1+\bar{w}_{\mathrm{de}}\right)\left[e^{\nu(r)}\right]^{-\frac{1+c_{s}^{2}}{2 c_{s}^{2}}}}{1+c_{s}^{2}}$,

where $e^{\nu(r)}=1-r_{g} / r$ outside the spherical object and $e^{\nu(r)}=(1-\alpha)^{3 / 2} /\left(1-\alpha r^{2} / R^{2}\right)^{1 / 2}$ inside it, where $\alpha \equiv$ $\equiv r_{g} / R$ and $R$ is the object radius. Such dependences for quintessential and phantom scalar field dark energies with different values of the effective sound speed

ISSN 2071-0194. Ukr. J. Phys. 2019. Vol. 64, No. 11 are shown in the left panel of Fig. 4. So, the density of dark energy deviates gradually from $\bar{\rho}_{\text {de }}$, when we approach the spherical static object. The character (increasing or decreasing) and rate of change depend on the type of dark energy (quintessence or phantom), parameters of the background dark energy, $c_{s}^{2}$ and $\bar{w}_{\text {de }}$, as well as on the gravitational radius of the central body. It should be noted that, as $c_{s}^{2} \rightarrow 0$, solution (11) loses its meaning. This means that the static solutions do not exist for dark energy with $c_{s}^{2}=0$ : it can only inflow (accretion) on a spherical static object or outflow from its neighborhood. One can see that the density of quintessential dark energy grows up with approaching the spherical static object and reaches the maximum value in the center, which is $\rho_{\mathrm{de}}(0) \approx$ $\approx \bar{\rho}_{\mathrm{de}}\left(1+\alpha\left(1+\bar{w}_{\mathrm{de}}\right) / c_{s}^{2}\right)$ for objects with $\alpha \ll 1$. The estimations of $\left(\rho_{\mathrm{de}}(0)-\bar{\rho}_{\mathrm{de}}\right) / \bar{\rho}_{\mathrm{de}}$ for planets, stars, galaxies, and clusters of galaxies show that it is very small $\sim 10^{-7}-10^{-10}$, if $c_{s}^{2} \sim 1$. In view of the smallness of the mean dark energy density in the galaxies and clusters of galaxies, we can conclude that the influence of dark energy with $c_{s}^{2} \sim 1$ on the structure of these objects or the dynamics of the other bodies in their gravitational field is negligible. On the contrary, the dark energy with $c_{s}^{2} \sim 0$ can agglomerate in the vicinity of a compact spherical object and affect its dynamics or orbits of their satellites. Thus, for such dark energy, we can expect observational manifestations on astrophysics scales. Their absence can give us the low limit for the value of $c_{s}^{2}$. Moreover, the scalar field 
dark energy with Lagrangian (2) and the EoS parameter (5) transform into scalar field dark matter energy with the satisfied conditions $\rho_{\text {de }}(r)+p_{\text {de }}(r)>0$ and $\rho_{\text {de }}(r)+3 p_{\text {de }}(r)>0$ in the dark energy dense regions with $\rho_{\text {de }}(r) \geq 3 \bar{\rho}_{\text {de }}\left(c_{s}^{2}-\bar{w}_{\text {de }}\right) /\left(1+3 c_{s}^{2}\right)$.

The ability of dynamic dark energy with $c_{s}^{2} \ll 1$ to change the shape and depth of the gravitational potential well of the compact stellar objects has been used in $[40,41]$ to establish the lower limit for the effective sound speed of dark energy on the basis of the accuracies of the elements of the orbits of all bodies in the Solar system within the orbit of Saturn, as well as the Chandrasekhar mass-radius relation for white dwarfs. Both approaches indicate that the effective speed of sound of dark energy is greater than $10^{-4}$ in the units of the speed of light.

Figure 4 (left panel) shows also that the density of phantom dark energy in the potential wells of the spherical static objects is lower than the background density and tends to zero (in the case of positive energy density), when the size of the object approaches the gravitational radius. This may mean that such phantom dark energy avoids neutron stars and black holes, $\rho_{\mathrm{de}} \rightarrow 0$.

\subsection{Stationary accretion onto a black hole}

Equation (11) shows also that the static solution does not exist for black holes, since the singularity is for $\rho_{\mathrm{de}}$ at $r=r_{g}$. So, the dynamical dark energy can inflow or outflow from the vicinities of black holes. Under the assumptions of test dark matter, which does not change the space-time metric, and a stationary dark energy flow, such problems were solved by different authors for different physical scenarios (see the recent review [42] and references therein).

The assumption of a stationary accretion of dark energy means that it inflows in the black hole vicinity, but its density and the flow velocity depend on the radial distance and do not depend on the time. In [12], we have analyzed the accretion of the scalar field dark energy with Lagrangian (2) and the EoS parameter (5) onto a black hole and have obtained the analytic solutions of the equations $T_{k ; i}^{i}=0$ for the density and the velocity of dark energy as a test component in the Schwarzschild field. The analytic radial dependences of the density $\rho_{\mathrm{de}}(r)$ and the 3 -velocity $v_{\mathrm{de}}(r)$ of dark energy are obtained for some special values of $c_{s}^{2}$. In the right panel of Fig. 4, the dependences of $\rho_{\text {de }}(r)$ for the quintessence $\left(w_{\infty}=-0.8\right)$ and phantom $\left(w_{\infty}=-1.2\right)$ scalar fields with effective sound speeds $c_{s}^{2}=1,2 / 3$, and $1 / 2$ are presented for the case of stationary accretion onto a black hole. They are quite similar to the static distributions of $\rho_{\text {de }}$ in the vicinity of a neutron star like the object with $r_{g} / R=0.6$, which is shown in the left panel of this figure. So, the above speculations about stationary distributions of dark energy in the vicinities of spherical static objects can be repeated for the distributions in the case of stationary accretion of test scalar field dark energy onto a black hole.

The important characteristic of the accretion of dark energy onto a black hole is the rate of change of the inflow mass of dark energy, which is, in our case, [12]

$\dot{m}=\pi \frac{\left(1+3 c_{s}^{2}\right)^{\frac{1+3 c_{s}^{2}}{2 c_{s}^{2}}}}{4 c_{s}^{3}}\left(1+w_{\infty}\right) \rho_{\infty} R_{g}^{2}$.

This shows that the quintessential scalar field dark energy inflows $(\dot{m}>0)$ onto a black hole, while the phantom one outflows $(\dot{m}<0)$. This can be easy understood in the framework of the Newtonian dynamics, if we take into account that the inertial mass of dark energy in the element of volume is $\propto\left(\rho_{\text {de }}+p_{\text {de }}\right)$ and is positive for the quintessential dark energy and negative for the phantom one. So, the quintessential scalar field is pulled into gravitational potential wells of static objects, while phantom field is pushed out of them. The direction of movement depends on the resulting force, which is the vector sum of the gravitation pulling/pushing force and the pressure gradient one. To evaluate the direction of the resulting force, one can calculate the right-hand side of Eq. (10) using Eq. (5) and the analytic solutions for $\rho_{\mathrm{de}}(r)$ for the stationary accretion onto a black hole presented in [12]. Everyone can see that the total force acting on the element of quintessential dark energy is directed to the center, while, for phantom one, it is directed from the center. This explains also avoiding the potential wells of astrophysical objects by the phantom dark energy. Equation (12) shows also that the mass of the quintessential dark energy accumulated onto a black hole is large for the model with a lower value of the effective sound speed. So, for some low value of $c_{s}^{2}$, the assumption about the test scalar field becomes wrong, and the backreaction of the inflowing matter on the space-time metric must be taken into account. Such models are most interesting, since

ISSN 2071-0194. Ukr. J. Phys. 2019. Vol. 64, No. 11 
they would have observational manifestations which can be used for the unveiling of the nature of dark energy.

1. D. Huterer, M.S. Turner. Prospects for probing the dark energy via supernova distance measurements. Phys. Rev. $D$, 60, 081301 (1999).

2. S. Perlmutter, et al. Discovery of a supernova explosion at half the age of the Universe. Nature 391, 51 (1998).

3. A. Riess, et al. Observational evidence from supernovae for an accelerating universe and a cosmological constant, Astron. J. 16, 1009 (1998).

4. B. Schmidt, et al. The High-Z Supernova search: Measuring cosmic deceleration and global curvature of the universe using type IA Supernovae, Astrophys. J. 507, 46 (1998).

5. Special issue on dark energy. Edited by G. Ellis, H. Nicolai, R. Durrer, R. Maartens. General Relativ. Gravit, 40, 219 (2008).

6. L. Amendola, S. Tsujikawa. Dark Energy: Theory and Observations (Cambridge Univ. Press, 2010).

7. Lectures on Cosmology: Accelerated Expansion of the Universe. Edited by G. Wolschin (Springer, 2010).

8. Dark Energy: Observational and Theoretical Approaches. Edited by P. Ruiz-Lapuente (Cambridge Univ. Press, 2010).

9. B. Novosyadlyj, V. Pelykh, Yu. Shtanov, F. Zhuk. Dark Energy: Observational Evidence and Theoretical Models. Edited by V. Shulga (Akademperiodyka, 2013).

10. B. Novosyadlyj et al. Do the cosmological observational data prefer phantom dark energy? Phys. Rev. D 86, 083008 (2012).

11. B. Novosyadlyj et al. Quintessence versus phantom dark energy: the arbitrating power of current and future observations. J. Cosmol. Astropart. Phys. 06, 042 (2013).

12. B. Novosyadlyj et al. Dynamics of dark energy in the gravitational fields of matter inhomogeneities. Phys. Rev. D 90, 063004 (2014).

13. R.R. Caldwell et al. Phantom energy: Dark energy with causes a cosmic doomsday. Phys. Rev. Lett. 91, 071301 (2003).

14. S.M. Carroll, M. Hoffman, M. Trodden. Can the dark energy equation-of-state parameter $\mathrm{w}$ be less than -1 ? Phys. Rev. D 68, 023509 (2003).

15. J.M. Cline, S. Jeon, G.D. Moore. The phantom menaced: Constraints on lowenergy effective ghosts. Phys. Rev. D 70, 043543 (2004).

16. N. Arkani-Hamed, H.C. Cheng, M.A. Luty, S. Mukohyama. Ghost condensation and a consistent IR modification of gravity. J. High Energy Phys. 05, 074 (2004).

17. F. Piazza, S. Tsujikawa. Dilatonic ghost condensate as dark energy. J. Astropart. Phys. Cosmol. 07, 004 (2004).

18. C. Deffayet, O. Pujolas, I. Sawicki, A. Vikman. Imperfect dark energy from kinetic gravity braiding, J. Astropart. Phys. Cosmol. 10, 026 (2010).
19. O. Sergijenko, B. Novosyadlyj. Sound speed of scalar field dark energy: Weak effects and large uncertainties, Phys. Rev. D 91083007 (2015).

20. C.L. Bennett et al. Nine-year wilkinson microwave anisotropy probe (WMAP) observations: Final maps and results. Astrophys. J. Suppl. 208, 20 (2013).

21. Planck Collaboration: P.A.R. Ade et al. Planck 2015 results. XIII. Cosmological parameters. Astron. Astrophys. 594, A13 (2016).

22. A.G. Riess et al. A 3\% solution: Determination of the Hubble constant with the Hubble space telescope and wide field camera 3.Astrophys. J. 730, 119 (2011).

23. N. Padmanabhan et al. A 2 per cent distance to $z=0.35$ by reconstructing baryon acoustic oscillations - I. Methods and application to the Sloan Digital Sky Survey. Mon. Not. Roy. Astron. Soc. 427, 2132 (2012).

24. L. Anderson et al. The clustering of galaxies in the SDSSIII Baryon oscillation spectroscopic survey: Baryon acoustic oscillations in the data release 9 spectroscopic galaxy sample. Mon. Not. Roy. Astron. Soc. 427, 3435 (2012).

25. F. Beutler et al. The 6dF Galaxy Survey: Baryon acoustic oscillations and the local Hubble constant. Mon. Not. Roy. Astron. Soc. 416, 3017 (2011).

26. A. Conley et al. Supernova constraints and systematic uncertainties from the first three years of the supernova legacy surveySupernova constraints and systematic uncertainties from the first three years of the supernova legacy survey. Astrophys. J. Suppl. 192, 1 (2011).

27. N. Suzuki et al. The Hubble Space Telescope cluster supernova survey. V. Improving the dark-energy constraints above $z>1$ and building an early-type-hosted supernova sample. Astrophys. J. 746, 85 (2012).

28. A. Lewis, S. Bridle. Cosmological parameters from CMB and other data: A Monte Carlo approach. Phys. Rev. D 66, 103511 (2002).

29. J.-Q. Xia et al. Dark energy constraints after the new Planck data. Phys. Rev. D 88, 063501 (2013).

30. A. Rest et al. Cosmological constraints from measurements of type Ia supernovae discovered during the first $1.5 \mathrm{yr}$ of the Pan-STARRS1 survey. Astrophys. J. 795, 44 (2014).

31. C. Cheng, Q.-G. Huang. Dark side of the universe after Planck data. Phys. Rev. D 89, 043003 (2014).

32. D. Shafer, D. Huterer. Chasing the phantom: A closer look at type Ia supernovae and the dark energy equation of state Phys. Rev. D 89, 063510 (2014).

33. B. Novosyadlyj et al. Dynamics of minimally coupled dark energy in spherical halos of dark matter. Gen. Relat. Gravit. 48, 30 (2016).

34. B. Novosyadlyj et al. Evolution of density and velocity profiles of dark matter and dark energy in spherical voids. Mon. Not. Roy. Astron. Soc. 465, 482 (2017).

35. B. Novosyadlyj et al. Voids in the Cosmic Web as a probe of dark energy. Condens. Matt. Phys. 20, 13901 (2017).

36. B. Novosyadlyj, V. Shulga, W. Han, Yu. Kulinich, M. Tsizh. Halos in Dark Ages: Formation and chemistry. Astrophys. J. 865, 38 (2018). 
37. R. Smith et al. Stable clustering, the halo model and nonlinear cosmological power spectra. Mon. Not. Roy. Astron. Soc. 341, 1311 (2003).

38. Yu. Kulinich et al. Nonlinear power spectra of dark and luminous matter in the halo model of structure formation. Phys. Rev. D 88, 103505 (2013).

39. B. Novosyadlyj et al. Gravitational stability of dark energy in galaxies and clusters of galaxies. Kinemat. Phys. Celest. Bodies 30, 53 (2014).

40. M. Tsizh et al. WDS'14 Proceedings of Contributed Papers - Physics, 21 (2014).

41. S. Smerechynskyi et al. submitted to Phys. Rev. D (2019).

42. E. Babichev et al. Black holes in the presence of dark energy. Usp. Fiz. Nauk 183, 1257 (2013).

Received 31.08.19
Б. Новосядлий

ТЕМНИЙ СЕКТОР В КОСМОЛОГІЇ:

ТЕМНА ЕНЕРГІЯ НА КОСМОЛОГІЧНИХ

I АСТРОФІЗИЧНИХ МАСШТАБАХ

$\mathrm{P}$ е $з$ ю м е

Обговорюються властивості та спостережні прояви динамічної темної енергії на космологічних й астрофізичних масштабах. Розглядається динамічна темна енергія у вигляді квінтесенційних та фантомних скалярних полів з різними значеннями параметра рівняння стану та ефективної швидкості звуку. Аналізується еволюція динамічної темної енергіі і тї вплив на динаміку розширення Всесвіту, формування гало і великих порожнин, їі поведінка в статичному світі галактик, в околицях зірок та чорних дір. Розглянуто можливі випробування для встановлення природи темної енергї. 